\title{
Four Categories of Support when Guiding Young Children in Managing their Upsets: An Interdisciplinary Exploration of the Literature
}

\author{
Douglas D. Bell, Jr., Ph.D. \\ Kennesaw State University
}

\begin{abstract}
Young children in early learning settings experience upset as part of their daily interactions with others and their environment. Young children with challenging behaviors are also on the rise in early learning classrooms. As young children navigate their responses to the way the world interacts with them, they rely on sensitive and trained adults to guide them in developing social and emotional abilities that will lead to their being productive members of society. This support in social emotional learning also increases the likelihood of the children learning in other areas and domains. Insight can be gained from the literature in a variety of disciplines to guide the teacher in early learning programs to scaffold the children in managing their upsets. Some of the contributing disciplines include but are not limited to psychology, child development, anthropology, and special education. This paper provides a synthesis of the literature and research from a variety of disciplines in supporting children in managing their upsets through the categories of promoting calm, creating a caring community of learners and establishing relationships, guiding children with expectations and appropriate expression, and supporting children in self-management and conflict resolution with accountability. These topics will be discussed with attention to impact on early learning teaching and classroom practices.
\end{abstract}

Keywords: early childhood; challenging behaviors; social emotional learning; discipline; guidance; selfcontrol

\section{Introduction}

When working with young children in a variety of facets and settings, practitioners are faced with many challenges related to development. The wide array of contextual influences on the development of children in the early childhood stages can create the need for nuances in practice that the professionals must integrate in their service. Such contextual influences include poverty, family cohesiveness, child rearing practices, to name a few.

The two settings most examined in the world of young children are the early learning setting, or classroom, and the home. Young children spend most of their waking hours in these two settings in many cultures (Small, 1999). In many industrialized nations, the child care setting is where many young children spend their waking hours. In other societies, the main setting for caring for and teaching the children in the early childhood years would be the home. In the United States alone, over 11 million children have a child care 
arrangements outside the home, many of which are in early learning settings (Child Care Aware, ND). There are also studies that explore young children's development in the community and in the workplace of the parents. In the cultures who actively seek support from professionals in how to work with and raise young children, the home and the early learning classroom are the most common environments (Gordon, Colaner, Usdansky, \& Melgar, 2013).

There is a growing trend for caregivers and teachers of young children to seek support in the area of social emotional development; in particular challenging behaviors (Westling, 2009). Challenging behaviors are increasing in early childhood settings (Powell, Dunlap, \& Fox, 2006). More adults are expressing difficulty in knowing what to do for children who are exhibiting challenging behaviors, teacher or parent (Neece, Green, \& Baker, 2012; Stormont \& Young-Walker, 2016). Research tells us that adults in children's daily lives often are at a loss of what to do and experience high levels of stress themselves when the children exhibit challenging behaviors. This is especially true when children have meltdowns and temper tantrums. These intense upsets are common events with children who have challenging behaviors or autism (Lipsky \& Richards, 2009).

Upset is a term that can mean a variety of things. Young children demonstrate upset when something happens they do not like. As previously stated, the intense expression of upset is the most challenging for teachers and caregivers to deal with, also known as meltdowns or temper tantrums. In an intensive upset, young children can scream and cry, demonstrate physical aggression such as hitting, kicking, biting, dropping to the floor and refuse to move, and throwing objects. Children respond to upset in intense ways for a variety of reasons and each child has a differing set of needs to learn to manage their upsets (Laurent \& Gorman, 2018). This variety suggests that there is not a universal technique, strategy, or program that will be completely effective for all children. Adults working with young children tend to utilize the research in their respective fields of study to work with the challenging behavior.

Adults are often observed with children experiencing upset, encouraging the child to not have the upset (Bailey, 2015). It is helpful and more realistic for adults to realize that with young children upset happens. A young child experiencing upset is a natural phenomenon and needs support and guidance from an adult. Many times the adult feels inadequate and embarrassed and is not able to offer much support (Haney, Houser, \& Cullen, 2018). Society and cultural influences put pressure on adults to view upset as a negative thing. This can lead adults to encourage suppression of upset feelings and therefore reduces guidance for the child experiencing the upset. When adults view upsets in young children as an opportunity to teach a social-emotional skill, they may be in a better position to teach upset management to the young children.

There are several approaches to working with children in upset. Applied Behavior Analysis Model, or ABA, is one approach the field of psychology implements. The counseling industry utilizes a reflective therapy and social skills teaching approach. The field of early intervention encourages the use of Positive Behavioral Interventions and Supports, PBIS. In the field of child development the child guidance approach is implemented. In early education there are a variety of discipline models to implement. Anthropology offers many case studies and ethnographies to help us understand upset and parenting practices around the world. These are a small set of well known approaches for helping children learn to manage their upsets.

Each of the frameworks or approaches described previously are well documented in the research. However, each also is extremely complex and has many details and nuances that make them difficult to implement. This is especially true for teachers and caregivers of young children. Early childhood teachers and 
caregivers in the heat of the upset need training and support to implement support with ease at the appropriate stage of the upset (Ritblatt, Hokoda, \& Van Liew, 2017).

Observation reveals that upset has three main stages. The first stage is pre-upset or the antecedent stage. This stage is what happens before the upset.The second is the upset occurrence stage, when the upset event actually takes place. The third is the post-upset stage. This is the stage after the upset happens. The preupset stage is what happens prior to an upset occurring. This requires preventative strategies. A large number of approaches and programs emphasize the importance of prevention. There is a vast amount of focus, training, and energy in preventing upset with young children. Fewer approaches offer guidance for adults in supporting the child during the upset occurrence stage. Many of the approaches also describe ways to dialogue with a child once the child has finished the upset in the post upset stage.

The fields of psychology, sociology, anthropology, early education, and early intervention are but a handful of academic disciplines which explore development through the varieties of contexts young children are served in. These and other disciplines offer research to help understand effective ways to support the development of young children and the context of upsets.

This paper is proposing a comprehensive approach to supporting young children in managing their upsets using effective practices from multiple disciplines. This will be done using a broad framework of practices from the literature of several disciplines which could be applied in the early learning environment for caregivers and teachers. When possible, the initial or seminal works were explored so some of the references may be old. This was intentional to ensure the integrity of the model or message that can be applied to managing upsets.

This proposed interdisciplinary framework is designed to support in each stage of upset The preventative measures suggested by many approaches are helpful to caregivers and teachers. However, during the upset occurrence stage, often adults are left feeling lost and embarrassed as to what to do. Exploring multiple disciplines can increase the response repertoire for caregivers and teachers. The framework employs literature in four categories of supports. The first category is promoting composure and calm. The second category is using relationship as a component of intervention. The third category is using communication as an intervention. The final category is using teaching and correcting as an intervention for helping children manage their upsets.

Each category of support has two constructs. These two constructs are prevention and response. Remembering that upset occurs in observed stages, caregivers and teachers need guidance in supporting each child in the stage that presents itself for intervention. This approach of prevention and response will be helpful for adults that have to support children in the pre-upset stage, upset occurrence stage, or the postupset stage. Young children need adult support in each stage of upsets if they are going to be effective at managing them.

\section{Promoting composure and calm}

The first category of support adults can provide children when helping them to manage their upset is the area of promoting composure and calm. Many programs designed to help young children manage their upsets have de-escalation or calming as a major component. 
When examining the research related to calm and its role in supporting children in managing their upsets, there are two aspects to consider. The first and less obvious aspect is the adult using self-calming strategies. The second, and obvious more aspect is guiding the child in using self-calming strategies.

\section{Adult Calming}

The adult self-calming is a prerequisite to supporting the child in self calm. Before helping a child to utilize self-calming de-escalation strategies, the adult needs to be able to remain calm. Adults can both self-calm and share calm. When a child is experiencing an upset, the supporting adult has two choices: adopt the upset or share calm.

The idea of sharing calm may seem less than scientific, but there are a couple of areas of research that support this view. First the area of social learning theory suggests that when we provide a model for the children, they are more likely to follow it (Joseph \& Strain, 2003). Next, there is some areas of research that mention the transfer of energy and the use of mirror neurons that would suggest that when the adult in the situation uses self-calming strategies the child will follow (Winerman, 2005).

\section{Child Calming}

When children are in their upset, they often feel out of control and unsafe and they need someone to help them feel safe. A position of empathy on the part of the adult during a child upset can be extremely helpful for both parties (Rogers, 1951). When children are in their upset, they are unable to think clearly and operate from their fight or flight functions of the brain (Wolfgang, 2009). Calm allows them to move into more feeling and thinking brain.

There is a prevention and a response aspect to promoting calm. The prevention aspect refers to teaching children calming strategies. When the children are in an emotional state where learning is optimal and they are not upset, adults can take the time to teach children the strategies that would be helpful to calm and selfregulate.

Children need to be taught to recognize when they need to use calming strategies; the procedures for the calming strategies; and how to read and respond to coaching and cues from an adult or friend offering support when feeling upset. Children benefit from cues given by caring adults or friends who may be able to recognize the early stages of upset. The children can then respond to these cues and self-regulate and thus be more likely stay in some control of their upset and feel more successful.

\section{Self-Regulation Strategies for Calming and De-Escalation}

The literature of several disciplines describes self-calming and de-escalation strategies which would be helpful for both child and adult. These strategies can be used by the adult to self-calm prior to supporting through modeling for the child (Joseph \& Strain, 2003). The strategies can be helpful for the child to use to self-regulate when they feel an upset coming on or when in an upset. There are many strategies for calming and self-regulation that have been mentioned in the research to be helpful. Some of the strategies are listed below.

Calming space. An important part of helping children learn to calm is giving them space for it. When adults force children into dialogue about being upset when they do not want to engage, the upset can escalate. Having a space to which the child may safely retreat gives the child freedom and opportunity to regain selfcontrol using strategies they have been taught, without judgement or threat (Jones,1987). 
Breathing exercises. Breathing techniques are central to young children remaining or becoming calm (Jones, 1987; Jones 2007). Relaxing breathing techniques help the child to move out of fight or flight mode and into a mode where dialogue and thinking can be possible. When doing breathing strategies teachers can use visual reminders or agreed upon signals. The visuals or signals serve as reminders of how to do the breathing exercise. The child should do at least two repetitions where they fill their lungs about 20 percent with air and let it out slowly with a few seconds between breaths.

Providing caregivers and teachers with a repertoire of tools and strategies for guiding children in selfcalming will increase the likelihood that they will be used. When parents and caregivers understand when to teach and use calming strategies, the children benefit greatly.

\section{Relying on Relationship}

Children develop in the context of relationships (Center on the Developing Child, ND). Children with stable relationships have less of a stress response in a strange or challenging situation. Children who lack stable relationships with caregivers are more likely to engage in significant stress response.

\section{Attachment}

Attachment of child to caregiver or teacher can serve as a powerful buffer against upset and adversity (Lieberman, 2018). Children rely heavily on their attachments to primary caregivers to serve as a secure base from which they can learn and explore. This learning and exploration include social emotional discovery. The secure attachment is relied upon for a foundation of trust (Bowlby, 1982). The field of infant mental health works diligently to support and foster secure attachments between parents and young children because this builds resilience against toxic stress (Franke, 2014). This can also be used as a foundation for preventing upsets and trust for guidance through an upset. When secure attachment is in place, young children can trust the adult will be there emotionally to help guide them through the upset and will offer empathy and care. More precisely, the relationship will be able to recover from the upset without long term strain (Lieberman, 2018).

\section{Intentional Connection}

Implementing attachment and connection can easily be overlooked by caregivers and teachers. It is sometimes assumed that because the child is in the family or in the class group, attachment and emotional connection are inevitable or already present. When adults assume a connection is in place, the child often remains emotionally lost within the group.

Emotional connection is something that requires time, effort, ongoing maintenance (Ostrosky \& Jung, 2005). The best way to build and maintain connection to increase the likelihood of secure attachment is through spending untaxed time together engaging in mutually pleasurable or needed experiences. In this context, untaxed means that the experience is not meant to teach the child and it is not meant to correct the child. It is instead an experience where adults and children are getting to know each other and enjoying each other's company.

Adults intentionally including emotional connection requires careful thought and planning. An intense pattern of upset on the part of the child requires more frequent and intentional emotional connections with the child. When child upsets are less intense and frequent, emotional connection experiences can be reduced in frequency but are still needed on a regular basis to maintain the attachment. Conduct connection activites throughout the day, each day, when the child is alert and ready to engage. Powerful connection experiences 
include touch, eye contact, a fun situation, and complete emotional presence on the part of the players (Bailey, 2000).

A support approach which includes regular and carefully planned emotional connection experiences can both help prevent and reduce upsets, as well as increase the likelihood of the child trusting the adult to guide through the upset (Purvis, Cross, \& Pennings, 2011). Adults benefit because they become more attached to the child and are able to rely on the increased attachment to attribute empathy and care during support episodes.

\section{Communicating}

The literature in a variety of disciplines emphasize the need for communication to support children in managing their upsets. Adults use communication in all three of the observed stages of upset: pre-upset, upset occurrence, and post-upset. Thus, communication is both a prevention support and a response support. It is important to remember however, that when children are experiencing the upset occurrence stage, they may not be able to logically dialogue about their issue or problem solve.

\section{Empathy and Support}

Communicating with children views of empathy and support can be very helpful at the pre-upset stage and the upset occurrence stage. Adults can offer care and empathy in both verbal and nonverbal communication attempts. Sharing with the child that you understand what they are experiencing and reassuring that you are here for them are forms of communication that can be helpful during an upset occurrence (Rogers, 1951).

\section{Validate Emotions}

Communicating with children about their feelings related to an upset is mentioned in the literature from several disciplines when supporting children with upset. As a preventative measure, adults can teach children to recognize their feelings and use certain phrases to express them. As a responsive measure, adults can use identification of feelings and model feelings phrases to help children pinpoint what they are experiencing or just experienced (Joseph, Strain, \& Ostrosky, 2005). As children become more competent in communicating their emotions the reliance on upset episodes decreases (Gordan, 1989).

Teaching and supporting children in talking about their emotions requires several steps according to researchers in the area of challenging behaviors. First the child needs to learn to name the feeling or emotion. Second the children need to be able to identify the feelings in themselves and others. Finally, children need to understand ways that feelings and emotions can be expressed (Joseph, Strain, \& Ostrosky, 2005).

\section{Communication Strategies for Empathy and Emotional Support}

Research literature supports the use of specific communication strategies when adults are trying to offer support in communicating empathy and emotions for young children learning to manage their upsets.

Active listening. Active listening is a method of engaging in conversation and listening to others that shows the speaker that the receiver is engaged in the conversation. The use of active listing improves communication (McNaughton, Hamlin, McCarthy, Head-Reeves, \& Schreiner, 2008). Active listening also helps the listener understand the real message being given by the speaker. In active listening in the context where a child is trying to manage upsets, an adult will listen and mirror back the feelings the adult feels the child is expressing (Gordan, 1989). Active listening also employs non-verbal behaviors known as 
acknowledgements where adults show they understand through behaviors such as head nods. Additionally, at times verbal prompts are used to encourage communication known as door openers.

I-Messages. One useful strategy for communicating with children about feelings and to offer empathy is the I-Message (Gordan, 1989). I-Messages are a way to show assertiveness when an event stimulates an emotion or feelings. The I-Message contains the word I and demonstrates the emotional response or need based on a happening. For example, a child after a toy dispute might say, When the blocks are all being used, Ifeel frustrated because I want some too. When an I-Message is used, the child is empowered because they asserted their feeling or need and it can be used as a catalyst for problem solving and dialogue, rather than the child engaging in an upset occurrence.

\section{Communication of Needs and Wants}

Another area of communication identified by literature in several disciplines when helping children manage their upsets is the communication of needs and wants. When young children are able to communicate what they need or want, it can serve as a cue for adults that they need to guide the child in calming and problem solving, or it can be used by the child to begin the problem solving process (Price \& Steed, 2016). IMessages can be used by the child to express wants and needs. The important factor is the ensure the child feels heard and the adult may need to guide the child in the expression act itself (Ostrosky, Hemmeter, Murry, \& Cheatham, 2005).

Providing children with strategies to communicate about their feelings, wants, and needs empowers young children to express themselves in ways the decrease the need for upset. Adults equipped with strategies for promoting communication and understands the benefit of communication are in a better position to support the young child in managing upsets.

\section{Teaching and Correcting}

The fourth category of support for young children learning to manage their upsets can be classified as teaching and correcting. The literature suggested that sometimes children use their current practices in upset because they do not know what else to do, or the current practices may have gotten them something they desired (Coleman, Crosby, Irwin, Dennis, Simpson, \& Rose, 2012). These children need to learn appropriate ways to manage their upsets. Another suggestion by the literature is that perhaps the child has learned an inappropriate way to manage their upsets and need to learn new and appropriate ways.

The idea of teaching and correcting is done from the perspectives that upsets happen. It is unreasonable to expect children to suppress their upsets and emotions, but instead to learn better ways to manage them. When the goal is to teach, the requirement to extinguish feelings of upset can be subsided and instead the managing of upset can be approached as offering an acceptable workable behavior. We teach replacement behaviors and guide the child in appropriate expression and problem solving (Powell, Dunlap, \& Fox, 2006).

Teaching and correcting usually occur in the pre-upset stage and the post-upset stage. The children can respond during the upset occurrence stage if there is a strong connection to the caregiver or teacher offering the guidance, but it is important to remember that often during the upset, the child is not in an appropriate mindset for clear thinking or problem solving. Thus teaching and correcting can be both preventative and responsive in nature. It is preventative because the adult teaches the child the needed skills during a time that the child is not in upset. It is responsive because the adult guides the child in using the pre-taught skill 
during the upset, or after the upset can allow an opportunity to do it over to experience the feeling of success. The literature is consistent in the need to teach young children appropriate social skills and correcting social misunderstandings to support them in managing their upsets.

\section{Perspective taking and Empathy Training}

The literature in several disciplines suggests that teaching children to appropriately use perspective taking and empathy skills can improve their management of upsets (Price \& Steed, 2016). While developmentally young children can struggle with understanding the perspective of others, they still benefit from learning about it.

In order for a young child to be guided through understanding the perspective of another, they need to be able to think clearly. Thus, perspective taking and empathy taking are not very useful when children are in the upset occurrence stage. Instead, as a preventative measure, teachers can guide children in perspective taking and empathy in the pre-upset period best. In addition, adults can also use perspective and empathy guidance as a responsive measure in the post-upset stage.

Adults can guide young children in perspective taking by asking children what their friend thinks when an upset occurs, if the upset involved their friend. Another Strategy that is useful is to have the child look at their friends face and then describe how their friend feels after the episode. It is important to state here that adults should only involve their friends' thoughts and feelings for perspective and empathy if the friend was victimized in some way. It is never helpful to call the child's attention to negative feelings of onlookers during an upset. This typically increases or triggers the upset further.

\section{Problem Response Programs}

The disciplines of psychology and special education at times refer to social skill training programs to support the children learning skills that can be generalized to managing their upsets. Such programs or curriculums can be used to teach the children things such as social problem solving, recognizing the size of a problem, and choosing appropriate responses to problems.

These social skill curriculums are useful because they help children identify the problem they are having, determine the level of seriousness of the problem, and select from a menu of response choices (Kavale \& Mostert, 2004). The adult needs to be trained in the implementation of the programs. Some of these programs use arbitrary simulated experiences to initially learn the social skill and others are used when the child is experiencing the upset.

\section{Problem Solving}

Much of the literature across disciplines suggest value in social problem-solving strategies, or conflict resolution, as a means of support for children learning to manage their upsets. Teaching young children appropriate ways to respond to conflicts can help reduce the need of inappropriate ways of expressing upset (Westling, 2009). The appropriate time to teach and utilize problem solving is during the pre-upset stage or the post-upset stage. As a preventative measure, adults can teach the appropriate problem-solving strategies before upsets occur so that when they experience an upset, they may employ the problem-solving skills. It is also helpful for adults to give cues and support to employ a learned problem-solving strategy if they see cues that an upset is about to be triggered for a child. 
Some strategies which have been suggested to be appropriate for problem solving with young children is using puppets to resolve problems, have a space or table for children to routinely go and resolve conflict, or use an item such as a talking stick (or soft item to substitute a talking stick) so each child can take turns expressing their point of view related to upset. Teaching a model script can be useful so the child has the tools to state what they need to state and get their needs met. Adults should be close by when children are problem solving together (Powell, Dunlap, \& Fox, 2006).

\section{Do Over}

Giving young children the opportunity to repeat and experience using the appropriate way can be a powerful tool in helping the children feel successful in managing their own upsets. Doing an experience over occurs in the post-upset stage and is therefore a responsive strategy. The Do Over consists of a child remembering or being guided to understand a more appropriate choice for managing his or her upset (Bailey, 2015). The child is then given the opportunity to do the experience again, employing the desirable upset management skill. By doing the experience over, the child can witness and feel what it is like to employ the skill successfully and thereby be more likely to repeat and generalize the skill.

\section{Consequences}

The final strategy found in literature of several disciplines for supporting children in managing their upsets is the use of appropriate consequences. Children need support in making intentional choices and taking responsibility for those choices (Glasser, 1988). Implementation of consequences should only be utilized for choices children have control over and should match the severity of the choice respectively. A child should never receive negative consequences for experiencing feelings of upset.

\section{Conclusion}

Young children inevitably will experience upset and need the support of an adult to learn how to manage those upsets. The research literature from a large variety of disciplines give suggestions of ways to support children in learning to manage upsets. These strategies can be classified into four categories: promoting composure and calm; using relationship as a component of intervention; communication as an intervention; and using teaching and correcting as an intervention for helping children manage their upsets.

\section{References}

Bailey, B. (2000). I Love You Rituals. New York, NY: Harper Collins.

Bailey, B. (2015). Conscious Discipline, expanded and updated ed. Oviedo, FL: Loving Guidance.

Bierman, K., Nix, R., Greenberg, M., Blair, C., \& Domitrovich, C. (2008). Executive functions and school readiness intervention: Impact, moderation, and mediation in the Head Start REDI program. Development and Psychopathology, 20(3), 821-843. doi:10.1017/S0954579408000394

Bowlby, J. (1982). Attachment. New York, NY: Basic Books.

Center on the Developing Child (ND). In Brief: The impact of early adversity on children's development. Harvard University. Retrieved from https://46y5eh11fhgw3ve3ytpwxt9r-wpengine.netdna-ssl.com/wpcontent/uploads/2015/05/inbrief-adversity-1.pdf 
Child Care Aware. (ND). Child care settings. Retrieved from https://usa.childcareaware.org/familiesprograms/about-child-care/.

Coleman, J., Crosby, M., Irwin, H., Dennis, L., Simpson, C., \& Rose, C. (2012). Preventing challenging behaviors in preschool: Effective strategies for classroom teachers. Young Exceptional Children. Retrieved from https://journals.sagepub.com/doi/abs/10.1177/1096250612464641.

Franke, H. (2014). Toxic stress: Effects, prevention, and treatment. children. Children, 1, 3, 390-402.

Glasser, W. (1988). Choice Theory in the Classroom. New York, NY: Quill.

Gordan, R., Colaner, A., Usdansky, M., \& Melgar, C. (2013). Beyond an "either-or" approach to home and center based child care: Comparing children and families who combine care types with those who use just one. Early Childhood Research Quarterly, 28, 4: 918-935.

Gordan, T. (1989). Teaching children self-discipline--at home and at school (1st ed.). New York: Times Books.

Haney, J., Houser, L., \& Cullen, J. (2018). Parental perceptions and child emotional and behavioral problems in autism. Journal of Autism and Developmental Disorders, 48, 1, 12-27.

Joseph, G., \& Strain, P. (2003). Helping young children control anger and handle disappointment. Young Exceptional Children, 7, 1, 21-29.

Joseph, G., Strain, P., \& Ostrosky, M. (2005). Fostering emotional literacy in young children: Labeling emotions. Center on the Social Emotional Foundations for Early Learning What Works Brief. Retrieved April 2019 from http://csefel.vanderbilt.edu/briefs/wwb21.pdf.

Jones, F. (1987). Positive Classroom Discipline. New York, NY: McGraw-Hill Books.

Jones, F. (2007). Tools for teaching, 2nd ed. Santa Cruz, CA: Fredric H. Jones \& Associates.

Kavale, K., \& Mostert, M. (2004). Social skills interventions for individuals with learning disabilities. Learning Disabilities Quarterly, 27, 31-43.

Laurent, A., \& Gorman, K. (2018). Development of emotion self-regulation among young children with autism spectrum disorders: The role of parents. Journal of Autism and Developmental Disorders, 48, 12491260.

Lieberman, A. (2018). The Emotional Live of the Toddler, revised ed. New York, NY: Simon \& Schuster.

Lipsky, D., \& Richards, W. (2009). Managing meltdowns: Using the SCARED calming technique with children and adults with autism. London: Jesscal Kingsley Publishers.

McNaughton, D., Hamlin, D., McCarthy, J., Head-Reeves, D, \& Schreiner, M. (2008). Learning to listen: Teaching an active listening strategy to preservice education professionals. Topics in Early Childhood Special Education, 27,4, 223-231.

Neece, C., Green, S., \& Baker, B. (2012). Parenting stress and child behavior problems: A transactional relationship across time. American Journal on Intellectual and Developmental Disabilities, 117, 1, 48-66. 
Ostrosky, M., Hemmeter, M., Murry, J., \& Cheatham, G. (2005). Helping children express their needs. Center on the Social and Emotional Foundations for Early Learning What Works Briefs

Ostrosky, M., \& Jung, E. (2005). Building positive teacher-child relationships. Center on the Social and Emotional Foundations for Early Learning What Works Briefs

Powell, D., Dunlap, G., \& Fox, L. (2006). Prevention and intervention for the challenging behaviors of toddlers and preschoolers. Infants and Young Children, 19, 1, 25-35.

Price, C., \& Steed, L. (2016). Culturally responsive strategies to support young children with challenging behavior. Young Children, 7, 5, 36-43.

Purvis, K., Cross, D., \& Pennings, J. (2011). Trust-based relational intervention: Interactive principles for adopted children with special emotional needs. Journal of Humanistic Counseling, Education, and Development, 48, 1, retrieved April 2019, https://doi.org/10.1002/j.2161-1939.2009.tb00064.x

Ritblatt, S., Hokoda, A., \& Van Liew, C. (2017). Investing in the early childhood mental health workforce development: enhancing professionals' competencies to support emotion and behavior regulation in young children. Brain Sciences. Retreived from https://doi.org/10.3390/brainsci7090120.

Rogers, C. (1951). Client-Centered Therapy. Boston, MA: Houghton Mifflin.

Small, M. (1999). Our Babies, Ourselves: How Biology and Culture Shape the Way We Parent. New York, NY: Anchor, Double Day Books.

Stormont, M., Young-Walker, L. (2016). Supporting professional needs for early childhood teachers: an exploratory analysis of teacher perceptions of stress and challenging behavior. International Journal on Disability and Human Development, 16, 1, 99-104.

Westling, D. (2009). Teachers and challenging behavior: Knowledge, views, and practices. Remedial and Special Education, 31, 1, 48-63.

Winerman, L. (2005). The mind's mirror: A new type of neuron, called a mirror neuron, could help explain how we learn through mimicry and why we empathize with others. Monitor on Psychology, 36, 9, 48.

Wolfgang, C. (2009). Solving Discipline and Classroom Management Problems. Methods and Models for Today's Teachers, $7^{\text {th }} e d$. Hoboken, NJ: Wiley \& Sons. 